\title{
Magnetostructural coupling from competing magnetic and chemical bonding effects
}

\author{
Joshua D. Bocarsly $\odot,{ }^{1, *}$ M. D. Johannes $\odot,{ }^{2}$ Stephen D. Wilson, ${ }^{1}$ and Ram Seshadri ${ }^{1}$ \\ ${ }^{1}$ Materials Department and Materials Research Laboratory, University of California, Santa Barbara, California 93106, USA \\ ${ }^{2}$ Center for Computational Materials Science, Naval Research Laboratory Washington, D.C. 20375, USA
}

(Received 4 June 2020; revised 13 October 2020; accepted 11 November 2020; published 28 December 2020)

\begin{abstract}
An understanding of the origins of giant magnetostructural coupling is developed for the compound MnAs, a magnetic material that has served as a prototype for many promising technologies including caloric refrigeration, magnetic actuation, and spintronics. We demonstrate that strong coupling between magnetism and crystal structure arises from an orbital-specific competition between exchange energies and kinetic (bonding) energies and that thermally activated spin fluctuations drive the unusual first-order phase transition from high to low symmetry upon heating. The underlying mechanism raises the prospect of an exotic paramagnetic state featuring local fluctuations in atomic positions and bonding on the timescale of the moment fluctuations. The results should inform the design of new materials with enhanced magnetostructural coupling, found at the border between structural and magnetic stability.
\end{abstract}

DOI: 10.1103/PhysRevResearch.2.042048

Since the 1950s [1-6], MnAs has served as the prototypical material for strong coupling between magnetism and crystal structure. Upon heating, a dramatic first-order magnetostructural phase transition is observed at $T_{C}=318 \mathrm{~K}$, whereupon the ferromagnetic (FM) hexagonal structure transforms to a lower-symmetry paramagnetic (PM) orthorhombic structure with a $2.5 \%$ smaller unit-cell volume. As the temperature is further increased, the hexagonal structure is recovered via a continuous transition around $T_{t}=398 \mathrm{~K}$. The transition at $T_{C}$ is of great interest because it can be actuated by temperature, field, and mechanical stress. In particular, a moderate magnetic field can be used to drive giant entropy changes in MnAs, enabling magnetic refrigeration based on the magnetocaloric effect [7-9]. Other promising magnetocalorics, such as $\mathrm{Gd}_{5}(\mathrm{Si}, \mathrm{Ge})_{4}, \mathrm{MnFe}(\mathrm{P}, X)$, and doped $\mathrm{LaMnO}_{3}$ display similarly strong coupling [10-16]; however, the microscopic mechanism governing giant magnetostructural coupling in MnAs and related materials remains an open question.

In the ground-state structure of $\mathrm{MnAs}, \mathrm{Mn}$ is arranged on a hexagonal lattice [Fig. 1(a)], with large spacing (3.72 A) within the basal plane and short spacing (2.85 $\mathrm{A})$ perpendicular to the plane. Upon heating above $318 \mathrm{~K}$ or applying pressures above 2 kbars [17], first-order transitions to orthorhombic symmetry are observed [Fig. 1(b)]. In these transitions, alternating rows of Mn move towards each other within the plane to create $\mathrm{Mn}-\mathrm{Mn}$ zigzag chains with shorter contacts lengths ( $3.38 \AA$ at ambient pressure), whereas alternating rows of As atoms move a similar distance in the

\footnotetext{
*jdbocarsly@mrl.ucsb.edu
}

Published by the American Physical Society under the terms of the Creative Commons Attribution 4.0 International license. Further distribution of this work must maintain attribution to the author(s) and the published article's title, journal citation, and DOI. perpendicular direction. We refer to the magnitudes of these modes within the orthorhombic cell as $\delta_{\mathrm{Mn}}=\Delta \mathrm{Mn}_{z} / c$ and $\delta_{\mathrm{As}}=\Delta \mathrm{As}_{x} / a$, indicating deviations of the atoms' fractional coordinates from their ideal hexagonal positions. At $318 \mathrm{~K}$ and ambient pressure, $\delta_{\mathrm{Mn}}$ and $\delta_{\mathrm{As}}$ are 0.027 and 0.025 , respectively [18].

There have been numerous attempts to explain the behavior of MnAs [4-6,19-22]. Goodenough and co-workers proposed that the transitions are $\mathrm{Mn}^{3+}$ high-spin to low-spin transformations [6,17,23]. Conversely, Bean and Rodbell [4] and De Blois and Rodbell [5] proposed a phenomenological model where a first-order transition can be realized in a sufficiently compressible material with a strongly volumedependent magnetic exchange. More recent computational efforts have concluded that the orthorhombic structure above $T_{C}$ is actually antiferromangetic (AFM) with the Mn moments alternating direction along the zigzag chains $[C$-type AFM, Fig. 1(c)] [19,24-26]. However, neutron diffraction points to a PM state without long-range order [3], and paramagnetic scattering experiments $[27,28]$ and chemical substitution studies [29] indicate ferromagnetic correlations and a local moment magnitude that remains approximately constant across $T_{C}$. Furthermore, a variety of unusual physical properties reported in MnAs are largely unexplained, including the large amounts of local atomic disorder [30,31], anomalous elastic properties $[32,33]$, and extremely low thermal conductivity [34]. Microscopic understanding consistent with the experimental situation has, so far, clearly been missing.

Here, we use electronic structure calculations on ordered and disordered magnetic states to establish physical mechanisms for both the pressure-driven and temperature-driven phase transitions in MnAs. Both transitions are found to arise from orbital-specific competition between kinetic energy and different types of exchange energies: intra-atomic Hund's coupling for the pressure-driven transition and interatomic magnetic exchange for the temperature-driven transition. 
(a) hexagonal $P 6_{3} / m m c$
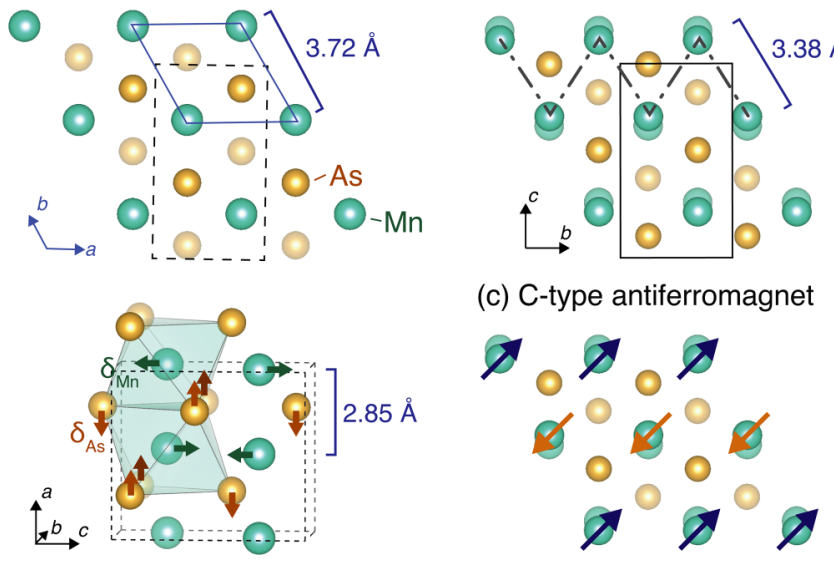

FIG. 1. Structure of MnAs in its (a) hexagonal and (b) orthorhombic forms. In (a), the orthohexagonal cell is shown with dashed lines and, at the bottom, the arrows indicate the directions of the atomic displacements that give rise to the orthorhombic structure. (c) The previously proposed $C$-type antiferromagnetic structure of the orthorhombic phase. All correlations out of the shown plane are ferromagnetic.

Furthermore, our calculations suggest that this competition may become dynamic in the paramagnetic phase as magnetic fluctuations couple with large fluctuations in interatomic spacing. This is expected to result in an unusual disordered phase, which can explain the anomalous elastic and transport behavior.

We began our investigation by performing density functional theory- (DFT-)based calculations [35-37] on MnAs Using the Perdew-Burke-Ernzerhof generalized gradient approximation revised for solids (PBEsol) [38]. Starting with the experimental ambient pressure hexagonal [39] and orthorhombic [18] structures, both with ferromagnetic moments, we fully relaxed the lattice parameters and internal atomic coordinates. This resulted in cells with volumes $13 \%$ and $19 \%$, respectively, smaller than experiment, in contrast with the typical slight overestimation of volumes often associated with the PBEsol approximation [40]. Additionally, the DFT energy of the orthorhombic cell was found to be lower than that of the hexagonal cell by $181 \mathrm{meV} \mathrm{f.u}^{-1}$, a result that is clearly unphysical given that the experimental ground state is ferromagnetic and hexagonal. Previous DFT studies have not addressed this inconsistency [25,41], presumably because a full relaxation into the global orthorhombic minimum was not investigated.

Employing the DFT $+U$ electron correlation correction via Dudarev's $U-J=U_{\text {eff }}$ formalism [42] localizes the Mn $d$ states that are systematically too diffuse in standard DFT [43] and brings both the lattice parameters and the magnetic moments $\left(3.4 \mu_{B}\right.$ per $\left.\mathrm{Mn}\right)$ of the hexagonal cell into good agreement with experiment for $U_{\text {eff }}=1.2 \mathrm{eV}$ with the PBEsol functional (Fig. S1 and S2 of the Supplemental Material [44]). Perhaps more importantly, this $U_{\text {eff }}$ proves to be the critical variable to correct the energetics, and the hexagonal cell is now $92 \mathrm{meVf.u} .^{-1}$ lower in energy than the orthorhombic one. We also tried calculations with the standard PBE functional, and found very similar results: Without
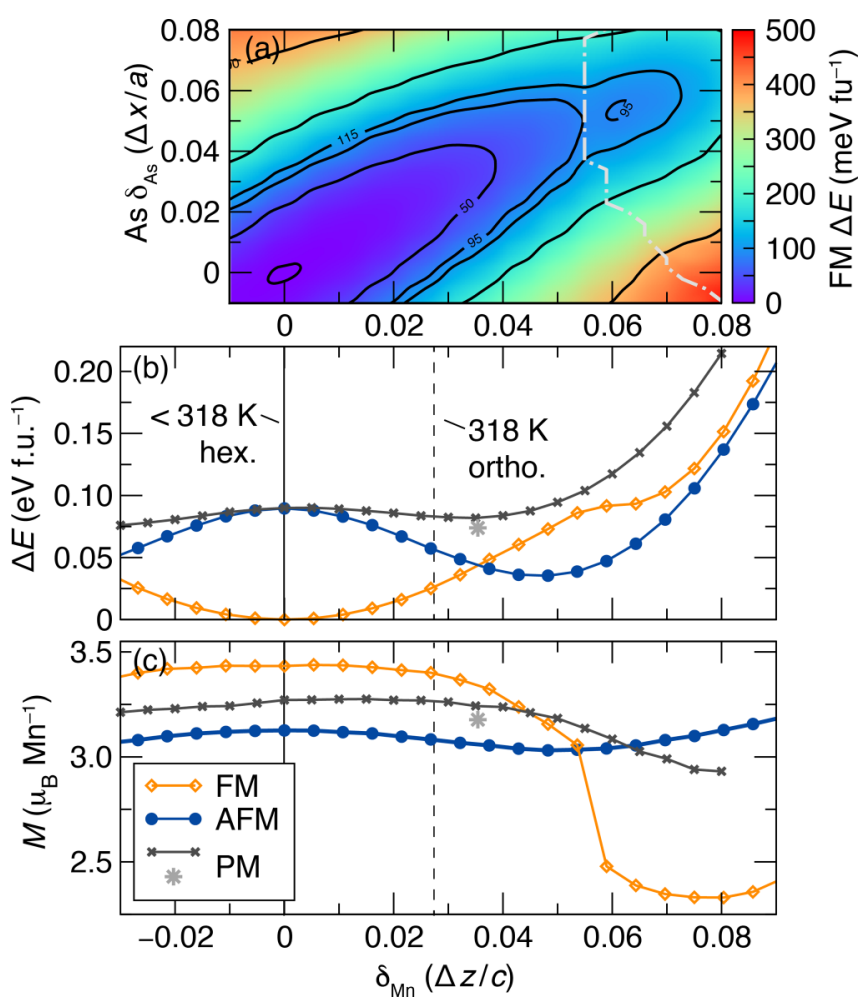

FIG. 2. (a) Two-dimensional (2D) energy surface of ferromagnetic MnAs as a function of Mn distortion and As distortion. (b) One-dimensional (1D) energy surfaces of FM, AFM, and PM MnAs as a function of Mn distortion. (c) Corresponding Mn local moment magnitudes.

$U$, the volumes are underestimated, and the ground state is incorrect. The energetics and volume, however, can be corrected with a small $U_{\text {eff }}=0.5 \mathrm{eV}$, which gives similar results to PBEsol with $U_{\text {eff }}=1.2 \mathrm{eV}$ (Fig. S3 of the Supplemental Material [44]).

Proceeding with PBEsol and $U_{\text {eff }}=1.2 \mathrm{eV}$, we found that the metastable orthorhombic state has a smaller Mn moment than the hexagonal ground state $\left(2.4 \mu_{B}\right.$ vs $\left.3.4 \mu_{B}\right)$. This moment and the DFT lattice parameters closely match (within $2 \%$ ) the experimental state obtained at low temperatures by applying pressure [45]. This indicates that the pressure-driven transition is consistent with a high-moment to low-moment transition. However, the volume of this orthorhombic cell is still $16 \%$ smaller than the ambient pressure orthorhombic cell observed at $318 \mathrm{~K}$, and the $\delta_{\mathrm{Mn}}$ is much larger $(0.061 \mathrm{vs}$ 0.027 ); therefore, this low-moment cell can only explain the pressure-driven transition, not the thermally driven transition.

We further investigated the energy surface of ferromagnetic MnAs during its transition from high-moment hexagonal to low-moment orthorhombic as a function of $\delta_{\mathrm{Mn}}$ and $\delta_{\mathrm{As}}$. Figure 2(a) shows a 2D energy surface created from 900 calculations where $\mathrm{Mn} z$ and As $x$ positions were held fixed, whereas all other coordinates and the lattice parameters were allowed to freely relax. We obtain a double-welled energy surface with local minima at the hexagonal structure $\left(\delta_{\mathrm{Mn}}=\right.$ $\left.0, \delta_{\mathrm{As}}=0\right)$ and in the low-moment orthorhombic structure $\left(\delta_{\mathrm{Mn}}=0.061, \delta_{\mathrm{As}}=0.053\right)$ with the barrier between these wells indicated with a white dashed line running principally 
vertically on the phase diagram. This analysis shows that the transition is driven by the Mn sublattice: Moving the $\mathrm{Mn}$ atoms without movement of the As atoms can traverse the phase transition line, but not vice versa. We, therefore, proceed to plot the 1D energy surface and local Mn moment as a function of $\delta_{\mathrm{Mn}}$ only [Figs. 2(b) and 2(c)] where the As $x$ position is now allowed to freely relax along with the other structural parameters. Here, a sharp drop in moment from $3.4 \mu_{B}$ to $2.4 \mu_{B}$ is observed as the hexagonal-to-orthorhombic phase boundary is crossed.

When we instead impose the $C$-type AFM structure, the energy surface looks very different. The potential is now single-welled, with an energy maximum at $\delta_{\mathrm{Mn}}=0$ and minimum at $\delta_{\mathrm{Mn}}=0.05$. Additionally, the local moment is virtually independent of $\delta_{\mathrm{Mn}}$. The magnetic stabilization energy (FM minus AFM), which is proportional to Mn-Mn magnetic exchange energy along the zigzag chain, is at its most negative at $\delta_{\mathrm{Mn}}=0$, and changes sign to positive (favoring AFM) for $\delta_{\mathrm{Mn}}>0.035$, consistent with a previous extended Heisenberg model investigation [41]. Notably, the experimental ambient-pressure $318 \mathrm{~K}$ orthorhombic cell has $\delta_{\mathrm{Mn}}=$ 0.027 , and $\delta_{\mathrm{Mn}}$ decreases as temperature rises [46]. Therefore, ambient-pressure MnAs always falls within the regime where ferromagnetic exchange dominates $\left(\delta_{\mathrm{Mn}}<0.035\right)$, corroborating the experimental observation that the paramagnetic correlations are ferromagnetic, not antiferromagnetic [27,28].

At ambient pressure and $318 \mathrm{~K}$, the observed $\delta_{\mathrm{Mn}}=0.027$ is close to the crossover in FM and AFM energies, consistent with the formation of a spin-fluctuation-based paramagnetic state. Since the dynamics of such a state are out of reach for DFT calculations, we simulate the paramagnetism using a special quasirandom structure (SQS) methodology [47], which has recently been applied successfully to paramagnets [48-51]. This approach involves constructing a supercell [we used the 96-atom supercell shown in Fig. 5(a)] and adjusting quasirandom up and down moments on the Mn atoms such that the local spin-spin correlations match the correlations of an infinite paramagnet (see the Supplemental Material [44] for details). When this disordered local moment cell is fully relaxed [gray star in Figs. 2(b) and 2(c)], it adopts an orthorhombic structure with the individual $\mathrm{Mn}$ and As atoms each relaxing differently in accordance with the broken local symmetries of the disordered cell. Overall, the average $\delta_{\mathrm{Mn}}$ is $0.035 \pm 0.006$. Even though this SQS calculation represents a static model for a dynamic phenomenon (paramagnetism), the agreement with experiment is remarkably good: The fully relaxed SQS cell has an average local magnetic moment of $3.2 \mu_{B}$ per $\mathrm{Mn}$, comparing well with $3.1 \mu_{B}$ from paramagnetic neutron scattering [27]. The relaxed lattice parameters are also all within $3 \%$ of their experimental $318-\mathrm{K}$ values [18] (Table S2 of the Supplemental Material [44]).

Figures 2(b) and 2(c) also show an energy surface for the disordered state, calculated using a smaller 64-atom SQS by fixing the $\delta_{\mathrm{Mn}}$ values but allowing all other structural degrees of freedom to relax. This surface shows a very shallow minimum around $\delta_{\mathrm{Mn}}=0.035$ and a maximum at $\delta_{\mathrm{Mn}}=0$. We note that, by construction, the SQS calculations give higher energies than the ordered states as the entropy which stabilizes the paramagnet is not considered in the calculated energy.

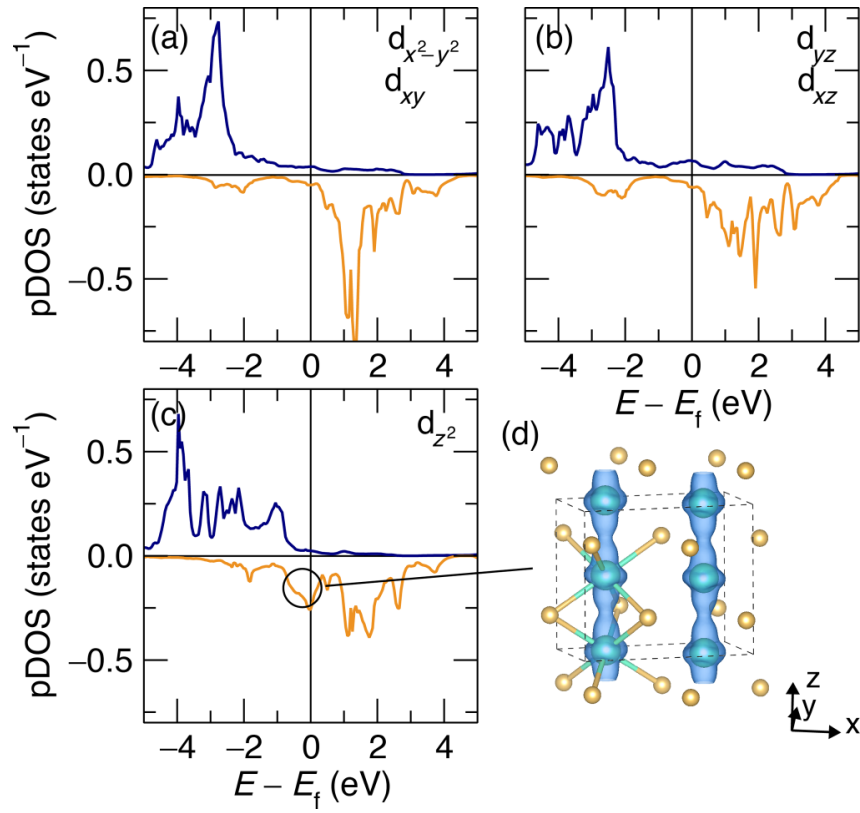

FIG. 3. (a)-(d) Projected Mn $d$-orbital partial densities of states for the ferromagnetic hexagonal structure, using a projection scheme oriented around the Mn-Mn contacts. Majority spin states are shown as positive density of states (DOS) values, and minority are shown as negative DOS values. (a) and (b) $d_{x^{2}-y^{2}}, d_{x y}, d_{y z}$, and $d_{x z}$ orbitals are all fully spin polarized, stabilized by Hund's coupling. (c) The $d_{z^{2}}$ orbital is more dispersive and is stabilized by Mn-Mn bonding as shown in (d) the partial charge density of the minority spin channel within $1 \mathrm{eV}$ below the Fermi level.

From these calculations, we can see that it is the highmoment ferromagnetism that establishes the high-symmetry lattice: Any pressure or temperature sufficient to disrupt this magnetic configuration towards PM, AFM, or low-moment FM states will simultaneously drive a structural distortion to orthorhombic. To understand the origins of this behavior, we investigated the orbital bonding properties of MnAs. Allthough $\mathrm{Mn}$ sits in a nearly perfect octahedron in the hexagonal structure, we found that the expected octahedral crystal-field splitting is absent from the electronic structure (Supplemental Material Fig. S5 [44]). This indicates that direct $\mathrm{Mn}-\mathrm{Mn}$ interactions beyond the immediate $\mathrm{Mn}-\mathrm{As}$ coordination shell play a significant role in the electronic structure, reminiscent of the FeAs family of superconductors which also exhibit strong and unusual magnetostructural couplings [52,53].

We, therefore, reoriented the $d$ orbital basis according to the Mn-Mn contacts as illustrated in Figs. 3(d) and Fig. 4(a). In this scheme, the $d_{z^{2}}$-orbital lobes point out of the plane, along the nearest-neighbor Mn-Mn contact, whereas one lobe of the $d_{x^{2}-y^{2}}$ orbital points along the Mn-Mn zigzag contact that is activated by the distortion. In the hexagonal structure, $d_{x^{2}-y^{2}}$ and $d_{x y}$ are degenerate, and $d_{y z}$ and $d_{x z}$ are degenerate. As shown in Fig. 3, all of the Mn $d$ orbitals except $d_{z^{2}}$ show partial DOS that are approximately half-filled with the majority spin states localized about $2.5 \mathrm{eV}$ below the Fermi level with unoccupied minority spin states above. This electronic structure is stabilized by Hund's coupling (i.e., intra-atomic 
ferromagnetic

(a)

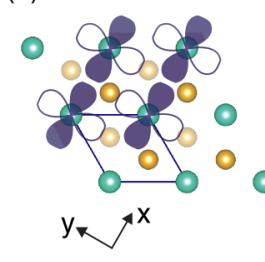

antiferromagnetic

(f)

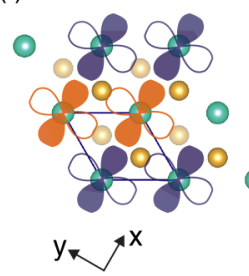

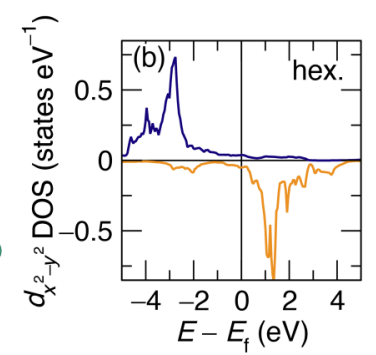

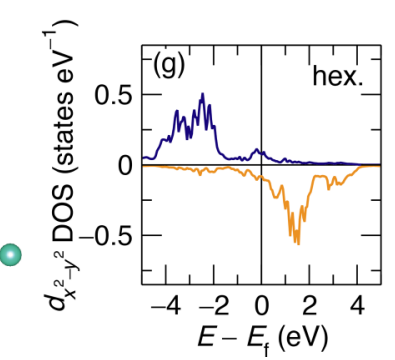

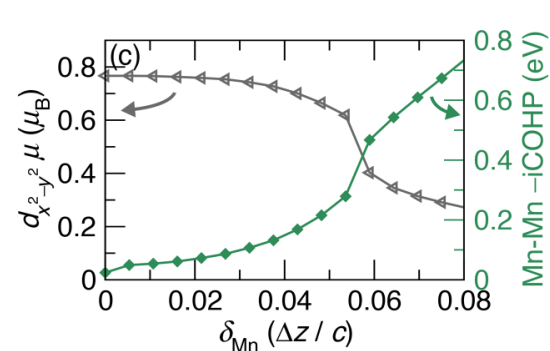
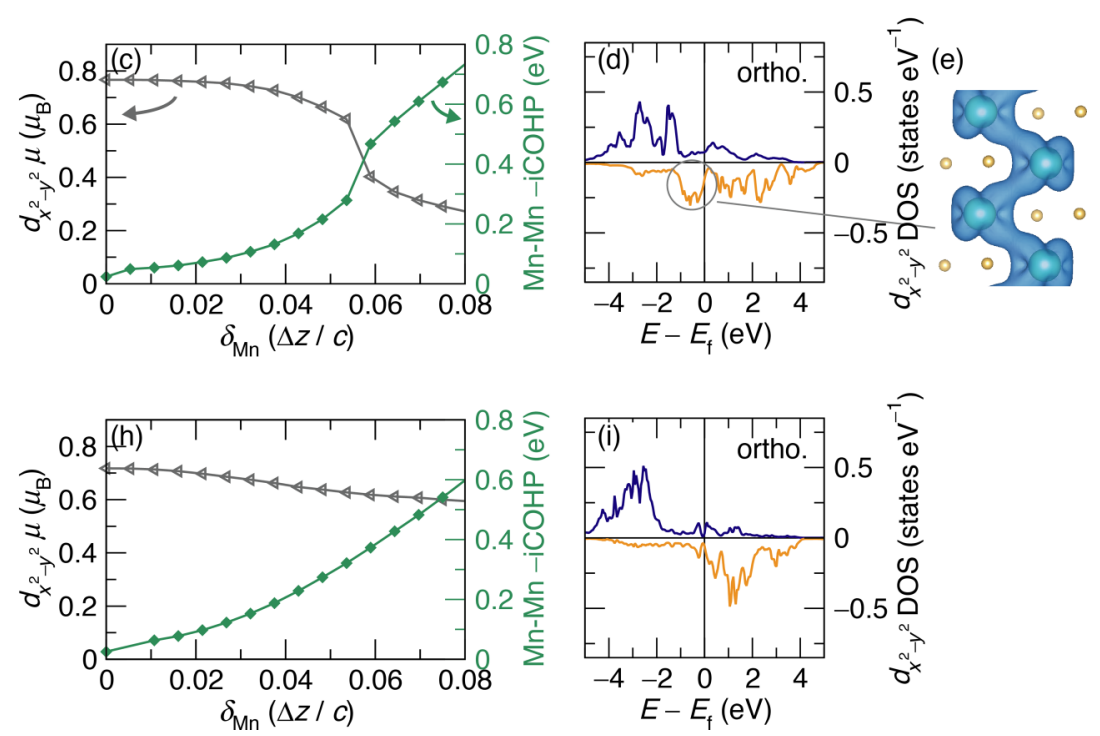

FIG. 4. Single-orbital properties of MnAs in the FM (a)-(e) and C-type AFM (f)-(i) structures. (a) Mn $d_{x^{2}-y^{2}}$ orbitals oriented along Mn-Mn contacts. (b) and (d) Projected partial $d_{x^{2}-y^{2}}$ DOS for the hexagonal and orthorhombic structures, respectively. (c) Projected $d_{x^{2}-y^{2}}$ moment and negative integrated crystal orbital Hamilton populations (-iCOHP) of the Mn-Mn zigzag contact as a function of Mn distortion. (e) Partial charge density of the minority spin channel within $1 \mathrm{eV}$ below the Fermi level for low-moment ferromagnetic orthorhombic MnAs. (f)-(i) show the same data as (a)-(e) except for the AFM case.

exchange), which is the driving force for the splitting of majority and minority spin states. Since only the majority spin states are occupied, this splitting lowers the energy. Conversely, the $d_{z^{2}}$ orbital shows a much more dispersive DOS consistent with a band formed by Mn-Mn bonding along the short contacts Fig. 3(d). For this orbital, it is the kinetic-energy benefit associated with chemical bonding that stabilizes the electronic structure.

In our projection scheme, the $d_{x^{2}-y^{2}}$ orbital shows large changes in the projected moment when a Mn distortion is introduced (Fig. 4.), whereas the other $d$ orbitals are comparatively unaffected (Fig. S6 of the Supplemental Material [44]). The Mn distortion forces $\mathrm{Mn}$ atoms together along the zigzag chains, causing neighboring $d_{x^{2}-y^{2}}$ orbitals to overlap. In the ferromagnetic case, this makes full spin-polarization of the $d_{x^{2}-y^{2}}$ untenable according to the Pauli exclusion principle, as electrons with the same spin cannot overlap spatially. This precipitates an electronic transition, seen in the reorganization of the DOS from a fully spin-polarized localized structure in Fig. 4(b) to a much more dispersive structure in Fig. 4(d). The new electronic structure loses Hund's coupling stabilization but partially compensates by forming Mn-Mn bonds along the zigzag chain [Fig. 4(e)]. The competition between Hund's coupling and bonding is clearly seen in Fig. 4(c), which shows the $d_{x^{2}-y^{2}}$ moment and the Mn-Mn zigzag contact bond strength (estimated using the integrated crystal orbital Hamilton population (-iCOHP) [54-58]) as a function of $\delta_{\mathrm{Mn}}$. As $\delta_{\mathrm{Mn}}$ increases, the moment drops precipitously, whereas the $-\mathrm{iCOHP}$ rises from 0 to $0.6 \mathrm{eV}$, indicating the formation of a $\mathrm{Mn}-\mathrm{Mn}$ bond. Furthermore, the orthorhombic distortion also increases the Mn-As and As-As -iCOHPs (Fig. S7 of the Supplemental Material [44]) as the asymmetric coordination optimizes bond distances. Altogether, these favorable bonding interactions explain the local stability of the low-moment orthorhombic state.
Therefore, we can see that the high-moment hexagonal to low-moment orthorhombic transition experienced by MnAs under pressure can be understood as arising from a competition between Hund's coupling and chemical bonding. At ambient pressure, Hund's coupling stabilization outweighs the kinetic-energy consideration. However, the application of pressure forces a reorganization of the electrons to comply with the Pauli principle, destabilizing the high-volume high-moment state in favor of a lower-moment more strongly bonded structure.

Even though the AFM state is never observed experimentally, it is informative to consider its behavior in order to understand how spin flips modify the system [Figs. 4(f)-4(i)]. In the AFM case, the Mn atoms that are brought together by the distortion have opposite spins and the Pauli exclusion principle does not come into play. As the $d_{x^{2}-y^{2}}$ orbitals begin to overlap, they are able to hybridize and form a metal-metal bond without disrupting the spin polarization. For this reason, the Mn local moment magnitudes remain approximately constant as a function of $\delta_{\mathrm{Mn}}$, and bonding and moment can all be simultaneously optimized at $\delta_{\mathrm{Mn}}=0.05$. However, this AFM state is not the ground state due to nonoptimized interatomic magnetic exchange [i.e., FM-AFM energy in Fig. 2(b)]. As discussed previously, this exchange is optimized at $\delta_{\mathrm{Mn}}=0$, stabilizing the FM hexagonal ground state.

Based on the analysis of the FM, AFM, and SQS states, we are now equipped to understand why the ambient-pressure transition from ferromagnetic to paramagnetic is coupled to a large structural distortion. The hexagonal ground state is not optimized from a bonding perspective but maximizes both interatomic magnetic exchange energy and Hund's coupling and is, therefore, lower in energy than all competing states. As temperature rises and spin-flip fluctuations begin to set in, the magnetic exchange energy benefit is weakened, and, at $318 \mathrm{~K}$, it becomes more favorable to structurally distort, forgoing the 


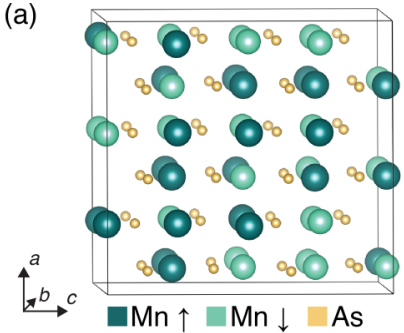

(b)

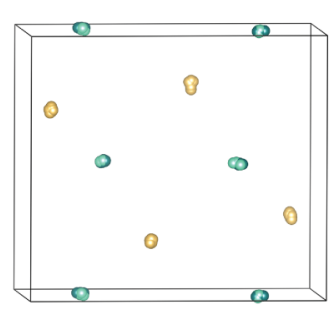

(c)

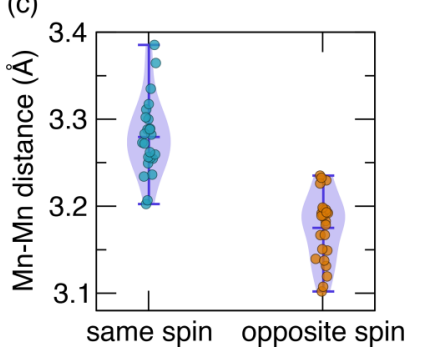

(d)

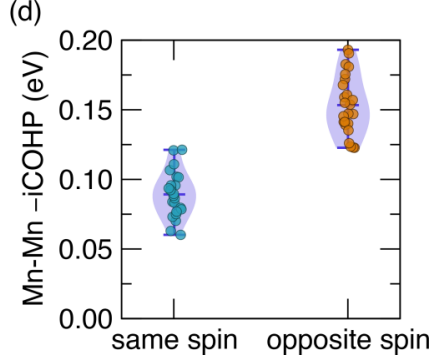

FIG. 5. (a) Structurally relaxed SQS used to simulate the paramagnetic state. (b) Projection of the supercell atomic positions into a single orthorhombic cell, showing $\mathrm{Mn}$ and As occupying a distribution of positions along the structural distortion modes. (c) Distributions of bond distance and (d) negative integrated crystal orbital Hamilton population for the 48 unique Mn-Mn zigzag contacts.

interatomic exchange energy in favor of the kinetic-energy (bonding) stabilization of the orthorhombic state. The spin fluctuations aid this transition by introducing AFM pairs of Mn atoms along the zigzag chains, allowing $d_{x^{2}-y^{2}}$ orbitals to partially maintain their Hund's coupling stabilization when distorting. Interestingly, all the competition takes place in the $\left(d_{x^{2}-y^{2}}\right)$-orbital channel. The small Mn-Mn contact length along the $d_{z^{2}}$ orbital causes it to remain in the bonded state at all temperatures, whereas long $\mathrm{Mn}-\mathrm{Mn}$ contacts along the other orbitals cause them to stay in their spin-polarized Hund's coupled states at all temperatures. Therefore, the key to this transition is the competition between exchange and bonding energies in half-filled Mn-Mn orbitals that are just on the verge of possible overlap.

This interplay among magnetic exchange, magnetic moment, and structural distortion has interesting implications for the paramagnetic state. In the fully relaxed SQS cell, we observed that the $\mathrm{Mn}$ and As atoms relaxed away from their symmetrical sites by up to $0.2 \AA$, leading to very different local environments for different atoms. Such large local distortions have not been seen in other paramagnets simulated using the SQS method [48-51]. Figure 5(a) shows the relaxed
SQS cell, and Fig. 5(b) shows all of the atoms from this supercell projected into a single primitive unit cell. Here, it can be seen that $\mathrm{Mn}$ and As atoms cluster into elongated clouds along the hexagonal-to-orthorhombic distortion modes; evidently, the PM state is most stable with a distribution of $\delta_{\mathrm{Mn}}$ and $\delta_{\mathrm{As}}$ values. In fact, this unusual finding can be connected to the mechanism of magnetostructural coupling described above. Figure 5 shows the Mn-Mn spacing (c) and bond strengths [-iCOHP, (d)] for each of the $48 \mathrm{Mn}-\mathrm{Mn}$ contacts along the zigzag chains in the SQS, plotted as a function of whether the pairs of atoms have the same spin direction or opposite spin direction. On average, the AFM pairs have bond distances $0.1 \AA(3 \%)$ shorter than FM pairs and bond strengths about twice as large. This is consistent with the idea that the Pauli exclusion principle disfavors $\left(d_{x^{2}-y^{2}}\right)$-orbital overlap in FM pairs of $\mathrm{Mn}$ atoms but not in AFM pairs. Importantly, the other Mn-Mn contacts in the structure, along other $d$ orbitals, show no dependence of the bond lengths or strengths on the spin correlation (Fig. S8 of the Supplemental Material [44]), highlighting once again that the competition-driven coupling occurs selectively in the $\mathrm{Mn} d_{x^{2}-y^{2}}$ orbitals.

The dependence of Mn-Mn bonding on local spin configuration implies that in the paramagnetic state of MnAs, thermal spin fluctuations can be expected to couple to large atomic displacements accompanied by the dynamic formation and breaking of metal-metal bonds. We predict this exotic disordered state should be detectable by large and dynamic local-symmetry breaking [30,31] as well as strong magnetovibrational inelastic neutron scattering, and anomalous thermal and spin transport. In particular, the local atomic disorder caused by this state may be invoked to explain the extremely low thermal conductivity [34] and the abnormal elastic properties [32,33]. Furthermore, the mechanisms for magnetostructural coupling driven by competition between exchange and kinetic-energy scales described here are expected to be a general feature of other systems displaying strong coupling, and similar features of the disordered magnetic states may be expected.

This work was supported by the National Science Foundation through Grant No. DMR-1710638. Partial support to S.D.W. through the NSF Materials Research Science and Engineering Center (Grant No. DMR-1720256, IRG-1) is acknowledged. M.D.J. acknowledges support from the Agile ICME Project from ONR Contract No. N0001418RX00212. Computational facilities were supported through NSF Grants No. CNS-1725797 and No. DMR-1720256 administered by the Center for Scientific Computing. J.D.B. was supported by the NSF Graduate Research Fellowship Program under Grant No. DGE-1650114. We thank Professor C. Van de Walle for helpful discussions.
[1] C. Guillaud, Les points de transformation des composés définis MnAs, MnBi en relation avec un mécanisme probable d'antiferromagnétisme, J. Phys. Radium 12, 223 (1951).
[2] B. T. M. Willis and H. P. Rooksby, Magnetic transitions and structural changes in hexagonal manganese compounds, Proc. Phys. Soc. London, Sect. B 67, 290 (1954). 
[3] G. E. Bacon and R. Street, Magnetic structure of manganese arsenide, Nature (London) 175, 518 (1955).

[4] C. P. Bean and D. S. Rodbell, Magnetic disorder as a first-order phase transformation, Phys. Rev. 126, 104 (1962).

[5] R. W. De Blois and D. S. Rodbell, Magnetic first-order phase transition in single-crystal MnAs, Phys. Rev. 130, 1347 (1963).

[6] J. B. Goodenough and J. A. Kafalas, High-pressure study of the first-order phase transition in MnAs, Phys. Rev. 157, 389 (1967).

[7] H. Wada and Y. Tanabe, Giant magnetocaloric effect of $\mathrm{MnAs}_{1-x} \mathrm{Sb}_{x}$, Appl. Phys. Lett. 79, 3302 (2001).

[8] V. K. Pecharsky and K. A. Gschneidner, Jr., Giant Magnetocaloric Effect in $\mathrm{Gd}_{5}(\mathrm{Si}, \mathrm{Ge})_{2}$, Phys. Rev. Lett. 78, 4494 (1997).

[9] V. Franco, J. Blázquez, J. Ipus, J. Law, L. Moreno-Ramírez, and A. Conde, Magnetocaloric effect: From materials research to refrigeration devices, Prog. Mater. Sci. 93, 112 (2018).

[10] W. Choe, V. K. Pecharsky, A. O. Pecharsky, K. A. Gschneidner, V. G. Young, and G. J. Miller, Making and Breaking Covalent Bonds Across the Magnetic Transition in the Giant Magnetocaloric Material $\mathrm{Gd}_{5}\left(\mathrm{Si}_{2} \mathrm{Ge}_{2}\right)$, Phys. Rev. Lett. 84, 4617 (2000).

[11] N. H. Dung, L. Zhang, Z. Q. Ou, and E. Brück, From first-order magneto-elastic to magneto-structural transition in $(\mathrm{Mn}, \mathrm{Fe})_{1.95} \mathrm{P}_{0.50} \mathrm{Si}_{0.50}$ compounds, Appl. Phys. Lett. 99, 092511 (2011).

[12] Z. B. Guo, Y. W. Du, J. S. Zhu, H. Huang, W. P. Ding, and D. Feng, Large Magnetic Entropy Change in Perovskite-Type Manganese Oxides, Phys. Rev. Lett. 78, 1142 (1997).

[13] M. E. Gruner, W. Keune, B. Roldan Cuenya, C. Weis, J. Landers, S. I. Makarov, D. Klar, M. Y. Hu, E. E. Alp, J. Zhao, M. Krautz, O. Gutfleisch, and H. Wende, Element-Resolved Thermodynamics of Magnetocaloric $\mathrm{LaFe}_{13-x} \mathrm{Si}_{x}$, Phys. Rev. Lett. 114, 057202 (2015).

[14] A. Barcza, Z. Gercsi, K. S. Knight, and K. G. Sandeman, Giant Magnetoelastic Coupling in a Metallic Helical Metamagnet, Phys. Rev. Lett. 104, 247202 (2010).

[15] J. D. Bocarsly, E. E. Levin, C. A. C. Garcia, K. Schwennicke, S. D. Wilson, and R. Seshadri, A Simple computational proxy for screening magnetocaloric compounds, Chem. Mater. 29, 1613 (2017).

[16] J. D. Bocarsly, E. E. Levin, S. A. Humphrey, T. Faske, W. Donner, S. D. Wilson, and R. Seshadri, Magnetostructural coupling drives magnetocaloric behavior: The case of $\mathrm{MnB}$ versus FeB, Chem. Mater. 31, 4873 (2019).

[17] N. Menyuk, J. A. Kafalas, K. Dwight, and J. B. Goodenough, Effects of pressure on the magnetic properties of MnAs, Phys. Rev. 177, 942 (1969).

[18] R. H. Wilson and J. S. Kasper, The crystal structure of MnAs above $40^{\circ} \mathrm{C}$, Acta Crystallogr. 17, 95 (1964).

[19] C. Kittel, Model of exchange-inversion magnetization, Phys. Rev. 120, 335 (1960).

[20] M. Shimizu, On the conditions of ferromagnetism by the band model, Proc. Phys. Soc. London 84, 397 (1964).

[21] S. K. Asadov, É. A. Zavadskî̂, V. I. Kamenev, E. P. Stefanovskiî, A. L. Sukstanskî̂, and B. M. Todris, Relation of magnetic and structural factors in the course of phase transitions in MnAs-based alloys, Phys. Solid State 42, 1696 (2000).
[22] V. I. Val'kov and A. V. Golovchan, Electronic mechanism of structural phase transitions in manganese arsenide, Low Temp. Phys. 30, 711 (2004).

[23] J. Mira, F. Rivadulla, J. Rivas, A. Fondado, T. Guidi, R. Caciuffo, F. Carsughi, P. G. Radaelli, and J. B. Goodenough, Structural Transformation Induced by Magnetic Field and "Colossal-Like" Magnetoresistance Response Above $313 \mathrm{~K}$ in MnAs, Phys. Rev. Lett. 90, 097203 (2003).

[24] M. K. Niranjan, B. R. Sahu, and L. Kleinman, Density functional determination of the magnetic state of $\beta$-MnAs, Phys. Rev. B 70, 180406(R) (2004).

[25] J. Łażewski, P. Piekarz, J. Toboła, B. Wiendlocha, P. T. Jochym, M. Sternik, and K. Parlinski, Phonon Mechanism of the Magnetostructural Phase Transition in MnAs, Phys. Rev. Lett. 104, 147205 (2010).

[26] J. Łażewski, P. Piekarz, and K. Parlinski, Mechanism of the phase transitions in MnAs, Phys. Rev. B 83, 054108 (2011).

[27] L. H. Schwartz, E. L. Hall, and G. P. Felcher, Magnetic structure of MnAs and $\mathrm{MnAs}_{0.92} \mathrm{P}_{0.08}$, J. Appl. Phys. 42, 1621 (1971).

[28] K. U. Neumann, K. R. Ziebeck, F. Jewiss, L. Däweritz, K. H Ploog, and A. Murani, Magnetic correlations in the paramagnetic phases of MnAs, Physica B 335, 34 (2003).

[29] K. Selte, A. Kjekshus, A. F. Andresen, and A. Zieba, On the magnetic properties of transition metal substituted MnAs, J. Phys. Chem. Solids 38, 719 (1977).

[30] G. A. Govor, Peculiarities of the first-order phase transition in MnAs, Phys. Status Solidi 90, K185 (1985).

[31] O. Palumbo, C. Castellano, A. Paolone, and R. Cantelli, Extended $\mathrm{x}$-ray absorption fine structure study of the MnAs local structure at the phase transitions, J. Phys.: Condens. Matter 17, 1537 (2005).

[32] M. Dörfler and K. Bärner, The elastic constants of MnAs, Phys. Status Solidi 17, 141 (1973).

[33] K. Bärner and H. Berg, Elastic anomalies at the second-order phase transition in MnAs, Phys. Status Solidi 49, 545 (1978).

[34] S. Fujieda, Y. Hasegawa, A. Fujita, and K. Fukamichi, Thermal transport properties of magnetic refrigerants $\mathrm{La}\left(\mathrm{Fe}_{x} \mathrm{Si}_{1-x}\right)_{13}$ and their hydrides, and $\mathrm{Gd}_{5} \mathrm{Si}_{2} \mathrm{Ge}_{2}$ and MnAs, J. Appl. Phys. 95, 2429 (2004).

[35] G. Kresse and J. Furthmüller, Efficient iterative schemes for $a b$ initio total-energy calculations using a plane-wave basis set, Phys. Rev. B 54, 11169 (1996).

[36] P. E. Blöchl, Projector augmented-wave method, Phys. Rev. B 50, 17953 (1994).

[37] G. Kresse and D. Joubert, From ultrasoft pseudopotentials to the projector augmented-wave method, Phys. Rev. B 59, 1758 (1999).

[38] J. P. Perdew, A. Ruzsinszky, G. I. Csonka, O. A. Vydrov, G. E. Scuseria, L. A. Constantin, X. Zhou, and K. Burke, Restoring the Density-Gradient Expansion for Exchange in Solids and Surfaces, Phys. Rev. Lett. 100, 136406 (2008).

[39] A. Zieba, K. Selte, A. Kjekshus, A. F. Andresen, O. Smidsrød, C.-O. Pontchour, P. Phavanantha, S. Pramatus, B. N. Cyvin, and S. J. Cyvin, Phase transitions in MnAs, Acta Chem. Scand. 32a, 173 (1978).

[40] P. Haas, F. Tran, and P. Blaha, Calculation of the lattice constant of solids with semilocal functionals, Phys. Rev. B 79, 085104 (2009).

[41] I. Rungger and S. Sanvito, Ab initio study of the magnetostructural properties of MnAs, Phys. Rev. B 74, 024429 (2006). 
[42] S. L. Dudarev, G. A. Botton, S. Y. Savrasov, C. J. Humphreys, and A. P. Sutton, Electron-energy-loss spectra and the structural stability of nickel oxide: An LSDA + $U$ study, Phys. Rev. B 57, 1505 (1998).

[43] V. I. Anisimov, J. Zaanen, and O. K. Andersen, Band theory and Mott insulators: Hubbard $U$ instead of Stoner I, Phys. Rev. B 44, 943 (1991).

[44] See Supplemental Material at http://link.aps.org/supplemental/ 10.1103/PhysRevResearch.2.042048 for details of the electronic structure calculation methods, comparison of experimental, and computational structures, and additional figures pertaining to the energy surfaces and orbital projections. The Supplemental Material refers to Refs. [59,60].

[45] V. P. Glazkov, D. P. Kozlenko, K. M. Podurets, B. N. Savenko, and V. A. Somenkov, Neutron diffraction investigation of the atomic and magnetic structures of MnAs at high pressures, Crystallogr. Rep. 48, 54 (2003).

[46] T. Suzuki and H. Ido, Relation between structural and magnetic properties of compound $\mathrm{MnAs}_{1-x} \mathrm{P}_{x}(0 \leqslant x \leqslant 0.275)$, J. Phys. Soc. Jpn. 51, 3149 (1982).

[47] A. Zunger, S.-H. Wei, L. G. Ferreira, and J. E. Bernard, Special Quasirandom Structures, Phys. Rev. Lett. 65, 353 (1990).

[48] B. Alling, T. Marten, and I. A. Abrikosov, Effect of magnetic disorder and strong electron correlations on the thermodynamics of CrN, Phys. Rev. B 82, 184430 (2010).

[49] P. Gorai, E. S. Toberer, and V. Stevanović, Thermoelectricity in transition metal compounds: The role of spin disorder, Phys. Chem. Chem. Phys. 18, 31777 (2016).

[50] G. Trimarchi, Z. Wang, and A. Zunger, Polymorphous band structure model of gapping in the antiferromagnetic and paramagnetic phases of the Mott insulators $\mathrm{MnO}, \mathrm{FeO}, \mathrm{CoO}$, and NiO, Phys. Rev. B 97, 035107 (2018).

[51] J. Varignon, M. Bibes, and A. Zunger, Origin of band gaps in 3d perovskite oxides, Nat. Commun. 10, 1658 (2019).
[52] D. J. Singh and M. H. Du, Density Functional Study of $\mathrm{LaFeAsO}_{1-x} \mathrm{~F}_{x}$ : A Low Carrier Density Superconductor Near Itinerant Magnetism, Phys. Rev. Lett. 100, 237003 (2008).

[53] D. A. Papaconstantopoulos, M. J. Mehl, and M. D. Johannes, Tight-binding Hamiltonian for LaOFeAs, Phys. Rev. B 82, 054503 (2010).

[54] R. Dronskowski and P. E. Bloechl, Crystal orbital Hamilton populations (COHP): Energy-resolved visualization of chemical bonding in solids based on density-functional calculations, J. Phys. Chem. 97, 8617 (1993).

[55] V. L. Deringer, A. L. Tchougréeff, and R. Dronskowski, Crystal Orbital Hamilton Population (COHP) analysis as projected from plane-wave basis sets, J. Phys. Chem. A 115, 5461 (2011).

[56] S. Maintz, V. L. Deringer, A. L. Tchougréeff, and R. Dronskowski, Analytic projection from plane-wave and PAW wavefunctions and application to chemical-bonding analysis in solids, J. Comput. Chem. 34, 2557 (2013).

[57] S. Maintz, V. L. Deringer, A. L. Tchougréeff, and R. Dronskowski, LOBSTER: A tool to extract chemical bonding from plane-wave based DFT, J. Comput. Chem. 37, 1030 (2016).

[58] S. Maintz, M. Esser, and R. Dronskowski, Efficient rotation of local basis functions using real spherical harmonics, Acta Phys. Pol., B 47, 1165 (2016).

[59] S. P. Ong, W. D. Richards, A. Jain, G. Hautier, M. Kocher, S. Cholia, D. Gunter, V. L. Chevrier, K. A. Persson, and G. Ceder, Python Materials Genomics (pymatgen): A robust, open-source python library for materials analysis, Comput. Mater. Sci. 68, 314 (2013).

[60] K. Momma and F. Izumi, VESTA 3 for three-dimensional visualization of crystal, volumetric and morphology data, J. Appl. Crystallogr. 44, 1272 (2011). 